\title{
Acquired Bilateral Central Vision Loss in a Young Adult Due to "Poppers Maculopathy"
}

\author{
Geoffrey Law ${ }^{1}$, Geoffrey Ching ${ }^{2}$, Gavin Docherty ${ }^{1}$, Eduardo Navajas ${ }^{1}$ \\ ${ }^{1}$ Department of Ophthalmology and Visual Sciences, University of British Columbia, Vancouver, BC, Canada \\ ${ }^{2}$ Faculty of Medicine, University of British Columbia, Vancouver, BC, Canada
}

\begin{abstract}
Purpose: To report a post-refractive surgery patient with central vision loss not correctable by refraction, subsequently found to have alkyl nitrite ("poppers") maculopathy.

Case report: A 35-year old male with a history of bilateral refractive surgery presented with sudden onset of bilateral central vision loss. His symptoms were unable to be corrected with refraction. On initial history, he denied the use of any medications or recreational drugs. He was referred for spectral domain optical coherence tomography (SD-OCT), which showed disruption of the foveal photoreceptor outer segments in both eyes. Upon further questioning, he admitted to the use of alkyl nitrites - colloquially known as "poppers" - 12 hours prior to the onset of visual symptoms. The patient was counselled on the guarded prognosis of this condition and was agreeable to ongoing follow-up with interval SD-OCT to monitor for potential resolution of the outer retinal changes.

Conclusion: A case of "poppers maculopathy" on a recent background of refractive surgery is presented, highlighting the importance of taking a detailed clinical and social history in patients with unexplained vision loss that cannot be improved with refraction.
\end{abstract}

Keywords: Post-refractive, Poppers maculopathy, Alkyl nitrite toxicity

\section{Introduction}

"Poppers" are recreational drugs that are inhaled to produce euphoria and muscle relaxation [1]. They have been available for several decades, with a side effect profile including hypotension, headache, and tachycardia. Curiously, the first case of popper-related maculopathy was only reported in 2004 [2]- since then, this disease has become increasingly diagnosed over the last decade [3]. In fact, recent data has shown that the lifetime usage of poppers is higher than expected with 5-6\% of French teenagers 6 and $60 \%$ of homosexual males in Australia admitting to having previously used alkyl nitrites [4]. This change in frequency is thought to be for several reasons, including improved detection by newer spectral domain optical coherence tomography (SD-OCT) technologies, recent changes in alkyl nitrite formulation from isobutyl to isopropyl nitrite, and the increased use of recreational drugs among the general population [4-7].
As this entity is becoming increasingly recognised, it is essential for the ophthalmologist to be familiar with this diagnosis as a potential mechanism of acquired vision loss. Herein, we describe a case of "poppers" maculopathy that presented to the emergency ophthalmology clinic at a quaternary hospital center.

Corresponding Author: Geoffrey Law, Department of Ophthalmology and Visual Sciences, Vancouver General Hospital Eye Care Centre, 2550 Willow Street Vancouver, BC, Canada Received date: July 29, 2019; Accepted date: August 06, 2019; Published date: August 07, 2019

DOI: https://doi.org/10.31546/JJOAR.1004 


\section{Case report}

A 35-year old male presented with a 2-day history of sudden onset, constant central blur in both eyes. He denied any other visual symptoms, including photopsias or metamorphosia. There was no history of trauma. He was otherwise healthy and endorsed a history of refractive surgery in both eyes 1 year ago. He denied the use of any medications or recreational drugs, including alcohol and tobacco. His best-corrected visual acuity (BCVA) was 6/7.5 OU. Anterior segment examination was unremarkable. Ophthalmoscopy showed a small yellow dot in the fovea of both eyes. SD-OCT of both eyes (Figure 1) demonstrated disruption of the foveal photoreceptor outer segments.
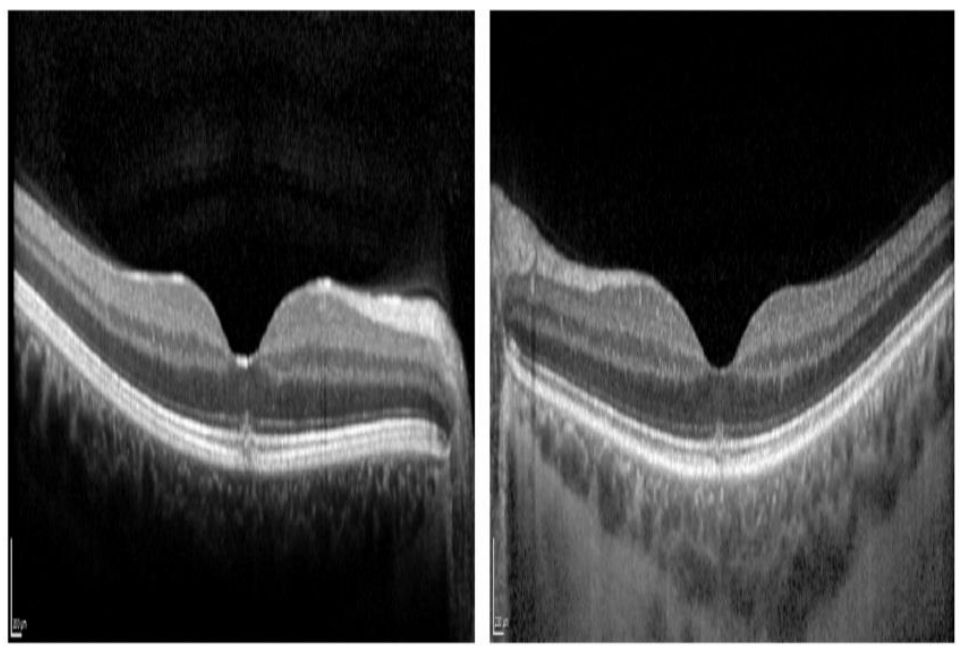

Figure 1: SD-OCT of both eyes

Upon further questioning of his recreational drug use, the patient subsequently admitted to the one-time use of "poppers", or alkyl nitrites, 12 hours before the onset of visual symptoms. This was his first time using the drug and he denied any other history of recreational substance use. Subsequent laboratory investigations did not uncover any other drug toxicity.

The patient was agreeable to regular follow-up with repeat SDOCT imaging in 2 months' time; he was also informed that his current symptoms were unrelated to his prior refractive surgery. A thorough discussion was had about the guarded visual prognosis of this condition and the patient was made aware that lutein has been associated with some visual recovery [1]. However; he elected not to start oral supplementation at this time.

\section{Discussion}

This case highlights the particular importance of taking a detailed social history in cases of unexplained visual loss that cannot be improved with refraction, as the diagnosis of "poppers" maculopathy could easily have been missed in this patient. The differential diagnosis of bilateral yellow spots in the fovea includes central serous retinopathy (CSR), bilateral stage 1 macular hole, and cystoid macular edema (CME). These can all effectively be ruled out by the SD-OCT in our patient, which did not demonstrate any evidence of subretinal or intraretinal fluid, or macular hole. Although the outer retinal disruption localized to the fovea can be caused by other conditions such as tamoxifen or solar retinopathy, the clinical history pointed strongly towards a diagnosis of "poppers" maculopathy.

To date, the exact mechanism of popper toxicity remains unknown. One thought is that alkyl nitrites cause upregulation of nitric oxide, which may increase photoreceptor photosensitivity $[1,4,5]$.This mechanism is corroborated by the fact that both nitrite toxicity and photic damage result in a maculopathy characterized by focal ellipsoid zone disruption or loss on SD-OCT [9].

There is no well-established treatment for alkyl nitrite maculopathy. Previous reports in the literature have documented spontaneous resolution of symptoms and imaging findings over time. Patients had better visual recovery if they abstained from the drug once symptoms began, or if they were taking the drug infrequently or for the first time [4,9]. Pahlitzsch et al. [10] described one patient who demonstrated near resolution of the maculopathy following 6 months of oral lutein supplementation. The time needed for full visual rehabilitation is highly variable, ranging from a period of 6 months to over 3 years. 8 Patients should be advised that the long-term prognosis of this disease remains largely uncertain.

Funding: This research did not receive any specific grant from funding agencies in the public, commercial, or not-for-profit sectors. 
Conflict of Interest: All authors have completed and submitted the ICMJE Form for Disclosure of Potential Conflicts of Interest. The authors have no proprietary or commercial interest in any of the materials discussed in this article.

Patient Consent: Written informed consent authorizing publication of anonymized clinical data and images was provided by the patient.

\section{References}

1. Docherty G, Eslami M, O'Donnell H. "Poppers Maculopathy": a case report and literature review. Can J Ophthalmol. 2018; 53:e154-e156.

2. Pece A, Patelli F, Milani P, Pierro L. Transient visual loss after amyl Isobutyl nitrite abuse. Semin Ophthalmol. 2004; 19:105-106.

3. Davies AJ, et al. The prevalence of visual symptoms in poppers users: a global survey. BMJ Open Ophthalmol. 2017; 1: e000015.

4. Krilis M, Thompson J, Atik A, Lusthaus J, Jankelowitz S. "Popper"-induced vision loss. Drug Alcohol Rev. 2013; 32:333-334.
5. Davies AJ, et al. Adverse ophthalmic reaction in poppers users: case series of "poppers maculopathy". Eye (Lond). 2012; 26:1479-1486.

6. Audo I, et al. Foveal damage in habitual poppers users. Arch Ophthalmol. 2011 Jun;129(6):703708.

7. Davies AJ, Kelly SP, Bhatt PR. "Poppers maculopathy"-an emerging ophthalmic reaction to recreational substance abuse. Eye (Lond) 2012; $26: 888$.

8. Fawcett RJ, Osborne NN. Flupirtine attenuates sodium nitroprusside-induced damage to retinal photoreceptors, in situ. Brain Res Bull. 2007; 73:278-288.

9. Rewbury R, Hughes E, Purbrick R, Prior S, Baron M. Poppers: legal highs with questionable contents? A case series of poppers maculopathy. Br J Ophthalmol. 2017; 101:1530-1534.

10. Pahlitzsch M, Mai C, Bergholz J, Bergholz R. Poppers maculopathy: complete restitution of macular changes in OCT after drug abstinence. Semin Ophthalmol. 2016; 31:479-484. 